\title{
Fauna e atributos microbiológicos de um Argissolo sob sistemas de cobertura no Sul do Brasil
}

\author{
Denice de Oliveira Almeida( ${ }^{(1)}$, Cimelio Bayer ${ }^{(2)}$ e Henrique Cesar Almeida(3)
}

\begin{abstract}
(1)Centro Universitário União Dinâmica das Cataratas, Rua Castelo Branco, no 349, Centro, CEP 85852-010 Foz do Iguaçu, PR, Brasil. E-mail: almeidadeni@yahoo.com.br (2)Universidade Federal do Rio Grande do Sul, Faculdade de Agronomia, Departamento de Solos, Avenida Bento Gonçalves, no 7.712, CEP 91540-000 Porto Alegre, RS, Brasil. E-mail: cimelio.bayer@ufrgs.br ${ }^{(3)}$ Universidade Federal da Integração LatinoAmericana, Instituto Latino Americano de Ciências da Vida e da Natureza, Avenida Tancredo Neves, № 6.731, CEP 85867-970 Foz do Iguaçu, PR, Brasil. E-mail: henrique.almeida@unila.edu.br
\end{abstract}

Resumo - O objetivo deste trabalho foi avaliar, em experimento de longa duração, a fauna edáfica e os atributos microbiológicos de um Argissolo Vermelho sob sistemas de cobertura em plantio direto. O trabalho foi realizado em Eldorado do Sul, RS, após 27 anos de implantação de experimento para avaliação dos seguintes sistemas de cultivo: A/M, aveia/milho; E/M, ervilhaca/milho; AE/M, aveia-preta + ervilhaca/milho; $\mathrm{AE} / \mathrm{MC}$, aveia-preta + ervilhaca/milho + feijão-caupi; e L/M, lablab/milho. Utilizou-se o delineamento de blocos ao acaso com três repetições. As amostragens da fauna edáfica, realizadas por armadilhas "pitfall", e dos atributos microbiológicos foram feitas no pós-manejo das coberturas, na primavera; durante o ciclo do milho, no verão; na pós-colheita do milho, no outono; e durante o ciclo das coberturas, no inverno. A inclusão de leguminosa em consórcio ou em sucessão de coberturas promoveu a ocorrência de organismos da fauna edáfica e aumentou $\mathrm{C}$ e $\mathrm{N}$ da biomassa microbiana, o que favoreceu o potencial de ciclagem e a disponibilidade de nutrientes. $\mathrm{O}$ sistema A/M de cobertura com gramíneas favoreceu as ordens Diptera, Hemiptera e Diplopoda. De forma geral, no plantio direto, os melhores sistemas de cobertura são os que incluem leguminosa.

Termos para indexação: bioindicadores, biomassa microbiana, consórcio de coberturas, organismos edáficos, plantio direto, qualidade do solo.

\section{Fauna and microbial attributes of an Ultisol under cover crop systems in the South of Brazil}

\begin{abstract}
The objective of this work was to evaluate, in a long-term experiment, the fauna and microbiological attributes of an Ultisol under no-tillage cover crop. The study was carried out in Eldorado do Sul, RS, Brazil, after 27 years of implantation of an experiment evaluating the following crop systems: $\mathrm{O} / \mathrm{C}$, oat/corn; $\mathrm{V} / \mathrm{C}$, vetch/corn; OV/C, oat + vetch/corn; OV/CP, oat + vetch/corn + cowpea; and L/M, lablab/corn. A randomized complete block design was used, with three replicates. Samplings of fauna, performed with pitfalls, and of microbiological attributes were done after cover crop management, in spring; during corn crop cycle, in summer; after corn crop harvest, in autumn; and during cover crop cycle, in winter. The inclusion of legume intercropped or in succession to cover crops promoted the occurrence of edaphic fauna and increased microbial biomass $\mathrm{C}$ and $\mathrm{N}$, which favored cycling potential and nutrient availability. The $\mathrm{O} / \mathrm{C}$ cover crop system with grasses favored the Diptera, Hemiptera, and Diplopoda orders. Overall, the best cover crop systems under notillage are those with legume inclusion.
\end{abstract}

Index terms: bioindicators, microbial biomass, cover crop intercropping, edaphic organisms, no-tillage, soil quality.

\section{Introdução}

Desde o princípio, o plantio direto preconiza mínimo revolvimento do solo, rotação de culturas e cobertura do solo com restos de matéria vegetal (Casão Junior et al., 2012). Os cultivos de cobertura do solo, visam, principalmente, a formação de matéria orgânica para proteção do solo (Harasim et al., 2016). Além disso, por oferecerem abrigo e alimento, as coberturas também atraem organismos edáficos (Rabary, 2008; Parra et al., 2009). No entanto, nas regiões de clima tropical, as maiores intensidade de chuva e temperaturas causam aumento na velocidade de decomposição das coberturas.

O planejamento da sucessão de culturas de cobertura, do tipo de cultura escolhido, e do sistema de 
cultivo (consórcio ou o cultivo solteiro) proporcionam diferentes intensidades de cobertura do solo, bem como diferentes teores nutricionais nas folhas e raízes dessas culturas. Dessa forma, esses cultivos, juntamente com as condições edafoclimáticas locais, podem resultar em combinações de riqueza e diversidade de organismos edáficos, com reflexos sobre a velocidade de decomposição dos resíduos e a ciclagem dos nutrientes no sistema solo-planta (Tiemann et al., 2015).

Trabalhos recentes mostram a importância da relação entre tipos de cobertura do solo e organismos edáficos. Baretta et al. (2014), ao avaliar um Latossolo Vermelho em Santa Catarina sob diferentes formas de manejo, observaram que o carbono da biomassa microbiana, o grupo taxonômico dos Collembolas, e os índices de diversidade e equabilidade destacamse como bioindicadores da qualidade do solo. Em experimento realizado em Argissolo VermelhoAmarelo com rotação de cultura, nos Estados Unidos, Tiemann et al. (2015) verificaram que a sustentabilidade da comunidade biológica do solo depende da quantidade e da diversidade química dos resíduos. Já, em Latossolo Vermelho, no Rio Grande do Sul, Silva et al. (2013) concluíram que o consórcio de culturas de cobertura aumentou a comunidade de Collembolas e a abundância de indivíduos, no início do crescimento das plantas de coberturas.

Assim, embora pouco conhecido no ambiente tropical do Sul do Brasil, o impacto de sistemas conservacionistas de manejo sobre a fauna e os microrganismos do solo é importante na ciclagem e na disponibilidade de nutrientes.

O objetivo deste trabalho foi avaliar, em experimento de longa duração (27 anos), a fauna edáfica e os atributos microbiológicos de um Argissolo Vermelho, sob diferentes cultivos de cobertura, em plantio direto.

\section{Material e Métodos}

O trabalho foi realizado na Estação Experimental Agronômica (EEA) da Universidade Federal do Rio Grande do Sul, em um experimento de manejo do solo com longa duração, conduzido desde 1983. A EEA está localizada no Município de Eldorado do Sul, RS

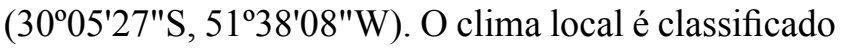
como subtropical úmido, Cfa de acordo com Köppen, e o solo, como Argissolo Vermelho distrófico típico (Santos et al., 2013), com a seguinte composição granulométrica: $220 \mathrm{~g} \mathrm{~kg}^{-1}$ de argila, $540 \mathrm{~g} \mathrm{~kg}^{-1}$ de areia e $240 \mathrm{~g} \mathrm{~kg}^{-1}$ de silte.

$\mathrm{O}$ experimento foi montado em sistema plantio direto, em delineamento experimental de blocos ao acaso, com parcelas de $5 \times 8 \mathrm{~m}$ e três repetições por tratamento. A cultura comercial utilizada foi o milho (Zea mays L.), combinado com diferentes coberturas, nos seguintes sistemas de cultivo: A/M, aveia-preta (Avena strigosa Schreb.) em cultivo solteiro no inverno/milho; E/M, ervilhaca (Vicia sativa L.) em cultivo solteiro no inverno/milho; AE/MC, aveia-preta + ervilhaca em consórcio no inverno e milho + feijãocaupi [Vigna unguiculata (L.) Walp] em consórcio no verão; $\mathrm{AE} / \mathrm{M}$, aveia-preta + ervilhaca em consórcio no inverno/milho; e L/M, cobertura de lablab (Dolichos lablab L.)/milho. Não foi aplicado nitrogênio mineral aos tratamentos avaliados.

As culturas de inverno foram manejadas com dessecação e passagem do rolo-faca, e a semeadura do milho foi feita em 15/12/2009 sobre esta palhada. A adubação foi realizada com $250 \mathrm{~kg} \mathrm{ha}^{-1}$ da fórmula N-P-K (00-20-20). Durante o desenvolvimento do milho, adotaram-se irrigação e controle de plantas daninhas, quando necessário. O rolo-faca foi passado novamente ao final da cultura do milho, depois da colheita. Após o ciclo do milho, sucedeu novo cultivo de culturas de cobertura de inverno, semeadas em $2 / 6 / 2010$. No cultivo solteiro, a aveia-preta foi semeada com $80 \mathrm{~kg}$ de semente por hectare e a ervilhaca com $70 \mathrm{~kg}$ de semente por hectare, enquanto, no consórcio, utilizaram-se 30 e $40 \mathrm{~kg} \mathrm{ha}^{-1}$ de aveia-preta e de ervilhaca, respectivamente. No consórcio do milho com o feijão-caupi, este foi semeado nas entrelinhas do milho em covas distantes cerca de $0,4 \mathrm{~m}$ uma da outra.

As coletas para análise da fauna e dos atributos microbiológicos do solo foram feitas nos seguintes períodos: no pós-manejo das coberturas de inverno, em 23/10/2009; durante o ciclo do milho, em 3/3/2010; na pós-colheita da cultura do milho, em 14/6/2010; e durante o ciclo das coberturas, em 6/10/2010.

A fauna do solo foi coletada por meio de armadilhas "pitfall”, confeccionadas por canos de PVC com 75 $\mathrm{mm}$ de diâmetro, permanentemente enterrados no solo. Fixaram-se três "pitfalls" por unidade experimental. Por ocasião das coletas, dentro dessas armadilhas, foram colocados recipientes de plástico que continham uma solução de etileno glicol (50\%) que ocupava $1 / 3$ do seu volume. Após cinco dias, os recipientes eram

Pesq. agropec. bras., Brasília, v.51, n.9, p.1140-1147, set. 2016 DOI: 10.1590/S0100-204X2016000900013 
coletados e a fauna classificada por lupa binocular para identificação dos organismos de acordo com suas classes, ordens ou família.

Com base na classificação da fauna edáfica, foram calculados: abundância de organismos, riqueza de táxons, índice de diversidade de Margalef, equabilidade de Pielou e dominância de Berger-Parker. $\mathrm{O}$ índice de diversidade de Margalef $(\mathrm{K})$ foi calculado pela fórmula: $\mathrm{K}=\mathrm{S}-1 / \log \mathrm{N}$, em que $\mathrm{S}$ é a riqueza de táxons e $\mathrm{N}$ é a abundância de organismos. A equabilidade de Pielou (e) foi determinada pela fórmula: $\mathrm{e}=\mathrm{H} / \operatorname{logS}$, em que $\mathrm{H}$ é o índice de Shannon, isto é, $\mathrm{H}=-\Sigma(\mathrm{pi} \log \mathrm{pi})$, com pi $=$ ni/S, em que ni é a densidade de cada grupo. Já a dominância de Berger-Parker (d) foi obtida pela fórmula: $\mathrm{d}=\mathrm{Nmáx} / \mathrm{N}$, em que Nmáx é a abundância máxima de um mesmo táxon.

As coletas de solo para análises microbiológicas foram realizadas com trado calador na profundidade de 0,0 a $0,1 \mathrm{~m}$, tendo-se retirado oito subamostras, que formaram uma amostra composta. As amostras foram passadas em peneiras com malha de $2 \mathrm{~mm}$ e mantidas em refrigeração, a $4^{\circ} \mathrm{C}$, até o momento da análise. As análises microbiológicas e químicas realizadas foram: carbono da biomassa microbiana (Cmic), nitrogênio da biomassa microbiana (Nmic), respiração basal microbiana $\left(\mathrm{CO}_{2}\right)$, quociente metabólico $\left(\mathrm{qCO}_{2}\right)$, enzimas urease e $\beta$-glucosidase, carbono orgânico (Corg), nitrogênio orgânico (Norg), emissão de óxido nitroso $\left(\mathrm{N}_{2} \mathrm{O}\right)$ e de amônia $\left(\mathrm{NH}_{3}\right)$, nitrato $\left(\mathrm{NO}_{3}{ }^{-}\right)$e amônio $\left(\mathrm{NH}_{4}^{+}\right)$.

A extração de carbono e nitrogênio da biomassa microbiana foi realizada por meio da metodologia de fumigação-extração descrita por Vance et al. (1987).

A respiração basal microbiana foi obtida pela incubação do solo, a $28^{\circ} \mathrm{C}$, por dez dias, segundo Jäggi (1976), enquanto a liberação de amônia foi avaliada por meio da incubação do solo, também a $28^{\circ} \mathrm{C}$, em presença de ácido bórico por 20 dias. Ao final do período, a amostra foi titulada com solução diluída de hidróxido de sódio de acordo com metodologia adaptada de Hernandes \& Cazetta (2001). O gás óxido nitroso foi coletado com uso de seringas de polipropileno, em câmaras estáticas fixas junto ao solo, e determinado por cromatografia gasosa.

A atividade total da enzima $\beta$-glucosidase foi obtida pela incubação do solo com o substrato enzimático $\rho$-nitrofenil- $\beta$-D-glucosideo, seguindo a metodologia de Eivazi \& Tabatabai (1988); já a atividade total da enzima urease foi determinada pela incubação do solo com substrato enzimático da ureia de acordo com Tabatabai \& Bremner (1972).

Os dados foram submetidos à análise multivariada de componentes principais (ACP); a significância das separações dadas pela ACP foi avaliada pelo teste de Hotelling, e as médias foram analisadas pelo teste LSD. O programa estatístico utilizado foi o Infostat, versão livre (Universidad Nacional de Córdoba, Córdoba, Argentina).

\section{Resultados e Discussão}

Os sistemas de culturas de cobertura estudados causaram modificação na fauna e nos atributos microbiológicos do solo, o que foi confirmado pela ACP. As coberturas com consórcio formaram um grupamento que proporcionou fauna distinta da dos cultivos solteiros, com variabilidade de $35 \%$ no CP1 e de $26 \%$ no CP2 (Figura 1 A). Porém, a cobertura com leguminosa se distinguiu dos sistemas de sucessão de gramíneas quanto aos atributos microbiológicos, com variabilidade de $68 \%$ no $\mathrm{CP} 1$ e de $24 \%$ no CP2 (Figura $1 \mathrm{~B}$ ).

Os sistemas $\mathrm{AE} / \mathrm{MC}$ e $\mathrm{AE} / \mathrm{M}$, com consórcio de coberturas do solo, estabeleceram oito associações entre os índices e os seguintes grupos taxonômicos da fauna edáfica (Figura $1 \mathrm{~A}$ ): Acarina, Isoptera, Isopoda, Collembola, Coleoptera, formas jovens, abundância e dominância, com destaque para número de indivíduos Isopoda e formas jovens no AE/M (Tabela 1). Ambos os sistemas de consorciação também promoveram maior concentração de Cmic, que foi $15 \%$ maior quando comparada à do $\mathrm{A} / \mathrm{M}$ (Tabela 2 ).

Os grupos taxonômicos Isopoda e formas jovens apresentaram preferências alimentares por altas quantidades de $\mathrm{N}$ no alimento. Cabe ressaltar que os Isopoda necessitam estabelecer associação com microrganismos, uma vez que não quebram algumas moléculas abundantes no tecido vegetal. Por esse motivo, preferem material orgânico que já recebeu primeiro ataque pelos microrganismos do solo (Correia et al., 2008). Desse modo, esse grupo parece ter sido sustentado pela maior quantidade de Cmic presente na matéria orgânica do tratamento AE/M.

$\mathrm{O}$ uso de sistemas de consórcio diversifica a composição vegetal da cobertura viva e os resíduos culturais do solo. Em relação à cobertura viva, o 
consórcio oferece maior densidade de cobertura do solo e, em relação aos resíduos culturais, disponibiliza maior diversificação de alimento à fauna edáfica e aos microrganismos por meio da variedade de propriedades químicas que proporciona (Sileshi et al., 2008). A fauna edáfica estabeleceu maior número de associações nos sistemas em consórcio do que nos cultivos solteiros, por estar diretamente sujeita tanto à cobertura viva quanto
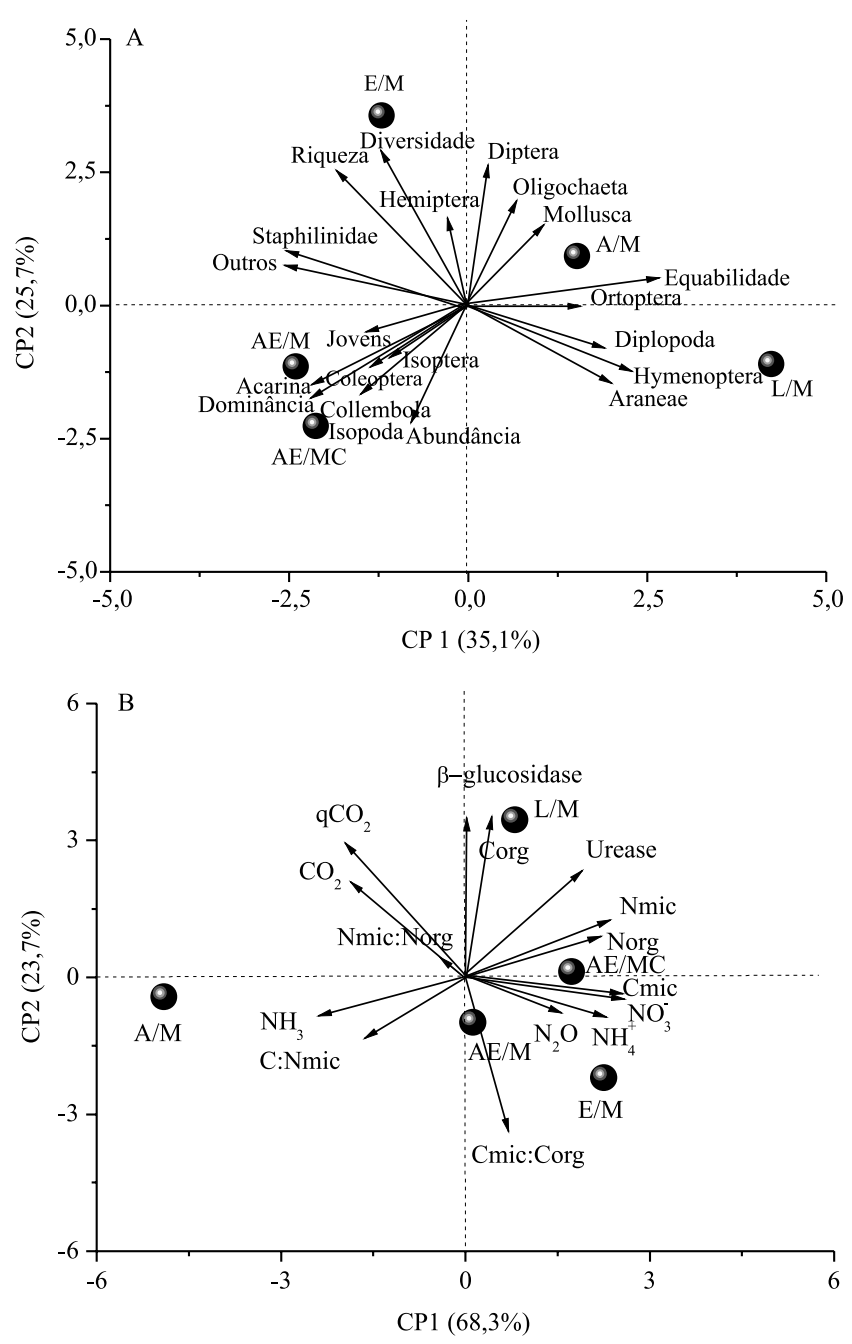

Figura 1. Componentes principais da fauna edáfica (A) e dos atributos microbiológicos (B) de um Argissolo Vermelho sob diferentes sistemas de cobertura, em plantio direto, em Eldorado do Sul, RS. E/M, ervilhaca (Vicia sativa)/milho (Zea mays); A/M, aveia-preta (Avena strigosa)/milho; L/M, lablab (Dolichos lablab)/milho; AE/MC, aveia-preta + ervilhaca/milho + feijão-caupi (Vigna unguiculata); e AE/M, aveia-preta + ervilhaca/milho. Corg, carbono orgânico; Norg, nitrogênio orgânico; Cmic, carbono microbiano; e Nmic, nitrogênio microbiano. aos resíduos promovidos pelo consórcio, que podem ser mais ou menos biodisponíveis à decomposição enzimática (Silva et al., 2010).

Efeitos positivos da diversidade de plantas sobre o Cmic são atribuídos, principalmente, à adição de leguminosa no consórcio (Milcu et al., 2008). Além disso, fontes de $\mathrm{C}$ e $\mathrm{N}$ encontradas em proporção variada, pela combinação de resíduos, como constatado no presente trabalho, contribuem para aumentar o Cmic (Sileshi et al., 2008; Silva et al., 2010), a resistência ao estresse e a homeostase do solo (Montecchia et al., 2011). Neste sentido, a fauna e os microrganismos do solo também atuam no controle biológico de doenças e pragas (Paul et al., 2015).

No consórcio, também há maior aproveitamento de espaço, ao se utilizar linhas e entrelinhas de cultivo, o que oportuniza plantas de maior porte, como a aveiapreta, e de menor porte como a ervilhaca. O consórcio entre gramíneas e leguminosas causa decomposição em tempo intermediário, por resultar em resíduos de relação $\mathrm{C} / \mathrm{N}$ mais adequados, em comparação aos dos cultivos solteiros (Ziech et al., 2015). Isso contribui para o aumento da disponibilidade de novos habitats favoráveis à colonização da fauna edáfica, o que beneficia a sustentabilidade ecológica dos sistemas de produção (Silva et al., 2007).

Os sistemas de culturas de cobertura com presença de planta leguminosa (AE/MC, $\mathrm{AE} / \mathrm{M}, \mathrm{E} / \mathrm{M}$ e $\mathrm{L} / \mathrm{M})$ estabeleceram relação com dez atributos microbiológicos: Cmic, Nmic, Corg, Norg, urease, $\beta$-glucosidase, $\mathrm{NH}_{4}{ }^{+}, \mathrm{NO}_{3}{ }^{-}, \mathrm{N}_{2} \mathrm{O}$ e Cmic:Corg (Figura 1 B). Na média de todos os tratamentos com a inclusão de uma leguminosa, foram verificados: $21 \%$ a mais de Norg, maior mineralização deste nutriente $\left(\mathrm{NH}_{4}{ }^{+} \mathrm{em}\right.$ $35 \%$ e $\mathrm{NO}_{3}^{-}$em $63 \%$ ), e $27 \%$ a mais de imobilização deste elemento na biomassa microbiana, quando comparado ao sistema A/M (Tabela 2).

A inclusão do cultivo de planta leguminosa no agroecossistema aumenta a fertilidade do solo por meio do incremento de $\mathrm{C} \mathrm{e} \mathrm{N}$, bem como da redução de perdas de nutrientes (Sileshi et al., 2008). O incremento em N oferecido pela leguminosa é importante para a fauna e os microrganismos, pois constitui importante fonte de nutrição para o crescimento, o desenvolvimento e a fecundidade dos animais (Parra et al., 2009). Já a biomassa microbiana forma um importante reservatório de elementos, especialmente $\mathrm{C}$ e N. Na forma de tecido microbiano, esses elementos estão temporariamente 
Tabela 1. Índices ecológicos e grupos taxonômicos da fauna edáfica em Argissolo Vermelho sob diferentes sistemas de cultivos de cobertura, em plantio direto ${ }^{(1)}$.

\begin{tabular}{|c|c|c|c|c|c|}
\hline \multirow[t]{2}{*}{ Fauna } & \multicolumn{5}{|c|}{ Sistemas de cultivos de cobertura } \\
\hline & $\mathrm{L} / \mathrm{M}$ & $\mathrm{AE} / \mathrm{MC}$ & $\mathrm{AE} / \mathrm{M}$ & $\mathrm{E} / \mathrm{M}$ & $\mathrm{A} / \mathrm{M}$ \\
\hline & \multicolumn{5}{|c|}{ Valores médios dos índices ecológicos } \\
\hline Abundância & $991^{\mathrm{ns}}$ & 1.151 & 1.089 & 935 & 1.123 \\
\hline Diversidade & $1,86 \mathrm{~b}$ & $1,94 a b$ & $1,96 a b$ & $2,16 \mathrm{a}$ & $1,98 \mathrm{ab}$ \\
\hline Dominância & 0,4 & 0,5 & 0,5 & 0,4 & 0,4 \\
\hline Equabilidade & $0,63 \mathrm{a}$ & $0,52 \mathrm{~b}$ & $0,53 \mathrm{~b}$ & $0,55 \mathrm{ab}$ & $0,58 \mathrm{ab}$ \\
\hline \multirow[t]{2}{*}{ Riqueza } & 9 & 10 & 10 & 10 & 10 \\
\hline & \multicolumn{5}{|c|}{ Número total de organismos Artrhopoda } \\
\hline \multicolumn{6}{|l|}{ Arachnida } \\
\hline Acarina & 143 & 226 & 186 & 168 & 161 \\
\hline Araneae & $98 \mathrm{a}$ & $60 \mathrm{ab}$ & $29 \mathrm{~b}$ & $39 \mathrm{~b}$ & $38 \mathrm{~b}$ \\
\hline \multicolumn{6}{|l|}{ Insecta } \\
\hline Coleoptera & 53 & 70 & 48 & 57 & 35 \\
\hline Díptera & $50 \mathrm{~b}$ & $47 \mathrm{~b}$ & $54 \mathrm{ab}$ & $87 \mathrm{ab}$ & $103 \mathrm{a}$ \\
\hline Hemíptera & $7 \mathrm{~b}$ & $15 \mathrm{~b}$ & $18 \mathrm{ab}$ & $22 \mathrm{ab}$ & $38 \mathrm{a}$ \\
\hline Hymenoptera & $154 \mathrm{a}$ & $106 \mathrm{ab}$ & $113 \mathrm{ab}$ & $85 \mathrm{~b}$ & $146 \mathrm{a}$ \\
\hline Orthoptera & 35 & 15 & 31 & 24 & 26 \\
\hline Staphylinidae & 6 & 8 & 9 & 9 & 7 \\
\hline Collembola & 350 & 515 & 470 & 364 & 480 \\
\hline \multicolumn{6}{|l|}{ Myriapoda } \\
\hline Diplopoda & $21 \mathrm{ab}$ & $15 \mathrm{abc}$ & $6 \mathrm{bc}$ & $4 \mathrm{c}$ & $28 \mathrm{a}$ \\
\hline \multicolumn{6}{|l|}{ Crustácea } \\
\hline Isopoda & $3 b$ & $8 \mathrm{~b}$ & $24 \mathrm{a}$ & $3 b$ & $4 \mathrm{~b}$ \\
\hline \multicolumn{6}{|c|}{ Número total de Mollusca } \\
\hline Gastropoda & 5 & 3 & 3 & 5 & 2 \\
\hline \multirow{3}{*}{ Oligochaeta } & & & total de & & \\
\hline & $4 \mathrm{ab}$ & $3 a b$ & $1 \mathrm{~b}$ & $6 \mathrm{a}$ & $2 b$ \\
\hline & \multicolumn{5}{|c|}{ Número total de organismos de outras classes } \\
\hline Formas jovens & $53 \mathrm{ab}$ & $51 \mathrm{ab}$ & $84 \mathrm{a}$ & $58 \mathrm{ab}$ & $44 \mathrm{~b}$ \\
\hline Outros & 9 & 9 & 13 & 4 & 9 \\
\hline
\end{tabular}

Tabela 2. Atributos microbiológicos de Argissolo Vermelho, sob diferentes sistemas de cobertura, em plantio direto ${ }^{(1)}$.

\begin{tabular}{|c|c|c|c|c|c|}
\hline \multirow[t]{2}{*}{ Atributos } & \multicolumn{5}{|c|}{ Sistemas de cultivos de cobertura } \\
\hline & $\mathrm{L} / \mathrm{M}$ & $\mathrm{AE} / \mathrm{MC}$ & $\mathrm{AE} / \mathrm{M}$ & $\mathrm{E} / \mathrm{M}$ & $\mathrm{A} / \mathrm{M}$ \\
\hline $\operatorname{Cmic}\left(\mu \mathrm{g} \mathrm{g}^{-1}\right)$ & $287 \mathrm{ab}$ & $291,9 a$ & $302,6 a$ & $283,8 \mathrm{ab}$ & $257,6 \mathrm{~b}$ \\
\hline Corg $\left(\mathrm{g} \mathrm{kg}^{-1}\right)$ & $16 \mathrm{a}$ & $13 \mathrm{~b}$ & $11 \mathrm{~cd}$ & $12 \mathrm{c}$ & $12 \mathrm{c}$ \\
\hline Cmic:Corg (\%) & $1,8 \mathrm{c}$ & $2,2 b$ & $2,7 \mathrm{a}$ & $2,3 b$ & $2,1 b$ \\
\hline $\mathrm{CO}_{2}\left(\mathrm{mg} \mathrm{g}^{-1} \mathrm{~h}^{-1}\right)$ & $2,2 \mathrm{a}$ & $1,6 b$ & $1,4 \mathrm{~b}$ & $1,3 b$ & $2,4 \mathrm{a}$ \\
\hline $\mathrm{qCO}_{2}^{(2)}$ & $7,9 \mathrm{a}$ & $2,8 \mathrm{~b}$ & $4,8 \mathrm{~b}$ & $4,9 b$ & $8,8 \mathrm{a}$ \\
\hline Urease $\left(\mu \mathrm{g} \mathrm{g}^{-1} 2 \mathrm{~h}^{-1}\right.$ de N-NH$\left.{ }_{4}^{+}\right)$ & $23,6 \mathrm{a}$ & $22,3 \mathrm{a}$ & $20,9 \mathrm{ab}$ & $21,8 \mathrm{ab}$ & $18,9 \mathrm{bc}$ \\
\hline$\beta$-glucosidase $\left(\mu \mathrm{g} \mathrm{g}^{-1} \mathrm{~h}^{-1}\right.$ de PNF) & $72,4 \mathrm{a}$ & $52,6 b$ & $47,7 b$ & $51,3 b$ & $53,5 b$ \\
\hline $\operatorname{Nmic}\left(\mu \mathrm{g} \mathrm{g}^{-1}\right)$ & $30,7 \mathrm{a}$ & $31,9 \mathrm{a}$ & $28,8 \mathrm{a}$ & $29,1 \mathrm{a}$ & $23,8 b$ \\
\hline $\operatorname{Norg}\left(\mathrm{g} \mathrm{kg}^{-1}\right)$ & $1,7 \mathrm{a}$ & $1,5 b$ & $1,7 \mathrm{a}$ & $1,4 \mathrm{bc}$ & $1,3 \mathrm{~cd}$ \\
\hline Nmic:Norg (\%) & 1,8 & 2,1 & 1,8 & 2,1 & 2,0 \\
\hline $\mathrm{NH}_{3}\left(\mathrm{~g} \mathrm{ha}^{-1} h^{-1}\right)$ & $128,6 \mathrm{c}$ & $183,8 b$ & $130,5 \mathrm{c}$ & $212,2 b$ & $297,2 \mathrm{a}$ \\
\hline $\mathrm{NH}_{4}^{+}\left(\mathrm{mg} \mathrm{g}^{-1}\right)$ & $10,7 \mathrm{ab}$ & $10,3 b c$ & $12,9 \mathrm{a}$ & $10,4 b c$ & $8,2 \mathrm{c}$ \\
\hline $\mathrm{NO}_{3}^{-}\left(\mathrm{mg} \mathrm{g}^{-1}\right)$ & $8,7 \mathrm{~b}$ & $8,8 \mathrm{~b}$ & $10,5 \mathrm{a}$ & $7,9 \mathrm{~b}$ & $5,5 \mathrm{c}$ \\
\hline $\mathrm{N}_{2} \mathrm{O}\left(\mu \mathrm{g} \mathrm{m}^{-2} \mathrm{~h}^{-1}\right)$ & $7,3 b$ & $48,7 \mathrm{a}$ & $22,3 b$ & $15,2 \mathrm{~b}$ & $4,5 b$ \\
\hline $\mathrm{C}:$ Nmic & $9,8 \mathrm{bc}$ & $9,3 \mathrm{c}$ & $10,5 \mathrm{bc}$ & $9,7 \mathrm{bc}$ & $10,8 \mathrm{ab}$ \\
\hline
\end{tabular}

${ }^{(1)}$ Médias seguidas de letras iguais, nas linhas, não diferem pelo teste $\mathrm{LSD}$, a $5 \%$ de probabilidade. ${ }^{(2)} \mathrm{qCO}$, expresso em $\mu \mathrm{g}$ de $\mathrm{C}-\mathrm{CO}_{2}$ por $\mu \mathrm{g}$ de $\mathrm{Cmic}$ por hora. Cmic, carbono microbiano; Corg, carbono orgânico; Nmic, nitrogênio microbiano; Norg, nitrogênio orgânico; e PNF, para-nitrofenol. L/M, lablab (Dolichos lablab)/milho (Zea mays); AE/MC, aveia-preta (Avena strigosa) + ervilhaca (Vicia sativa)/milho + feijão-caupi (Vigna unguiculata); AE/M, aveia-preta + ervilhaca/milho; E/M, ervilhaca/milho; e A/M, aveia-preta/milho. 
seguros contra perdas do agroecossistema, sendo liberados de forma mais lenta e gradual.

$\mathrm{O}$ sistema $\mathrm{E} / \mathrm{M}$ obteve maior índice de diversidade de Margalef e número de indivíduos do grupo Oligochaeta, em comparação ao $\mathrm{AE} / \mathrm{M}$ e ao $\mathrm{A} / \mathrm{M}$ (Tabela 1). Os Oligochaeta apresentam maior exigência na qualidade do material orgânico (Paul et al., 2015), e as leguminosas, na maior proporção do N. Esse grupo de organismos é fundamental para sequestro de $\mathrm{C}$ por meio da incorporação e da distribuição do material orgânico por todo o perfil, o que aumenta o contato deste com o solo (Cardoso et al., 2013). Além disso, atua na formação de agregados estáveis que protegem a matéria orgânica contra a rápida decomposição (Paul et al., 2015).

O sistema L/M promoveu, em média, aumento de $44 \%$ da atividade da enzima $\beta$-glucosidase e maior número de Araneae, quando comparado aos sistemas de cobertura AE/M, A/M e E/M (Tabelas 1 e 2). A arquitetura da cobertura vegetal viva, composta por uma cobertura arbustiva e densa, como é o caso do lablab, estabelece correlação positiva com a quantidade de Araneae (Mendes et al., 2011).

Os sistemas $\mathrm{L} / \mathrm{M}$ e $\mathrm{AE} / \mathrm{MC}$ promoveram acréscimo de $38 \%$ no $\mathrm{C}$ orgânico e de $25 \%$ na atividade da enzima urease, em comparação aos demais tratamentos (Tabela 2). Sabe-se que maiores conteúdos de Corg e Norg são capazes de sustentar maiores $\mathrm{C}$ e $\mathrm{N}$ microbianos e, consequentemente, síntese de enzimas do solo (Qin et al., 2010).

Entretanto, a combinação de coberturas $\mathrm{AE} / \mathrm{MC}$ ocasionou a maior perda de $\mathrm{N}$ por meio da emissão de óxido nitroso, $295 \%$ a mais do que as demais coberturas (Tabela 2). Apesar da maior perda de $\mathrm{N}_{2} \mathrm{O}$, a inclusão de uma leguminosa proporcionou maior acúmulo deste nutriente no tecido microbiano (Tabela 2). Em virtude da grande capacidade de fixação de $\mathrm{N}$ atmosférico, essas coberturas inserem grande quantidade deste elemento no solo, o que aumenta a atividade dos microrganismos (Torres et al., 2015).

Os sistemas $\mathrm{L} / \mathrm{M}$ e $\mathrm{A} / \mathrm{M}$ proporcionaram maior número de Hymenoptera (Tabela 1). Este grupo apresenta espécies fungívoras, detritívoras e predadoras, o que contribui ativamente para o equilíbrio dinâmico em sistemas conservacionistas. Os organismos predadores podem influenciar a riqueza e a diversidade das espécies, o que regula indivíduos abaixo da cadeia trófica (Bradford et al., 2007). Por sua vez, os detritívoros preparam o material orgânico para ser incorporado ao solo (Silva et al., 2006).

As coberturas dos sistemas $\mathrm{L} / \mathrm{M}$ e $\mathrm{A} / \mathrm{M}$ ainda proporcionaram cerca de $60 \%$ a mais de respiração basal microbiana e $100 \%$ a mais de metabolismo, em comparação às demais culturas de cobertura (Tabela 2). O metabolismo celular é dependente do estado fisiológico e é influenciado por diversos fatores. Um metabolismo mais alto pode indicar tanto um distúrbio ecológico quanto um alto nível de produtividade do agroecossistema (Silva et al., 2010). A maior respiração encontrada na leguminosa tropical pode estar ligada à maior produtividade proporcionada por esta cobertura, devido à sua ligação com as mais altas concentrações de Corg, Norg, Nmic, e atividade enzimática de $\beta$-glucosidase e urease (Figura $1 \mathrm{~B}$ e Tabela 2). De modo oposto, o maior metabolismo encontrado na cobertura de aveia-preta pode estar ligado a um distúrbio ecológico pela ligação desta cobertura com menores concentrações de Corg, Norg, Cmic, Nmic, e atividade enzimática de $\beta$-glucosidase e urease.

O sistema $\mathrm{A} / \mathrm{M}$ com uso de gramínea solteira como cobertura promoveu maior número de Diptera, Hemiptera e Diplopoda do que L/M e AE/MC (Tabela 1). As gramíneas oferecem maior potencial de proteção do solo a esses grupos, em razão da maior permanência dos resíduos sobre o solo. Entretanto, isso ocorre em detrimento das leguminosas, que, pela baixa relação $\mathrm{C} / \mathrm{N}$, apresentam rápida decomposição, o que deixa o solo desprotegido ainda no início do ciclo do milho (Ziech et al., 2015).

\section{Conclusões}

1. O plantio direto com espécies leguminosas como plantas cobertura, consórcio ou sucessão com outras espécies, favorecem a ocorrência de organismos da fauna edáfica e a atividade microbiana, o que promove a ciclagem do nitrogênio e do carbono no solo.

2. O plantio direto com cobertura de gramínea - milho no verão e trigo no inverno - favorece a ocorrência de grupos da fauna ligados à maior necessidade de abrigo, e promove a atividade microbiana relacionada a perdas de carbono e nitrogênio.

3. Os sistemas consórcio de aveia-preta (Avena strigosa) + ervilhaca (Vicia sativa), no inverno, e de milho (Zea mays) + feijão-caupi (Vigna unguiculata)

Pesq. agropec. bras., Brasília, v.51, n.9, p.1140-1147, set. 2016 DOI: 10.1590/S0100-204X2016000900013 
(AE/MC) ou de lablab e milho (L/M), no verão, favorecem o conteúdo de carbono orgânico do solo e a atividade da enzima urease.

4. O sistema aveia-preta/milho (A/M), com cultivo de cobertura com a gramínea solteira, promove os grupos taxonômicos Diptera, Hemiptera e Diplopoda; enquanto que os consórcios aveia-preta + ervilhaca (inverno)/milho (AE/M) e ervilhaca/milho (E/M) favorecem o grupo Hymenoptera; o L/M, o grupo Araneae; e o E/M, o grupo Oligochaeta.

5. De forma geral, os melhores sistemas de cobertura no plantio direto são os que incluem alguma espécie leguminosa.

\section{Referências}

BARETTA, D.; BARTZ, M.L.C.; FACHINI, I.; ANSELMI, R.; ZORTÉA, T.; BARETTA, C.R.D. Soil fauna and its relation with environmental variables in soil management systems. Revista Ciência Agronômica, v.45, p.871-879, 2014. DOI: 10.1590/ S1806-66902014000500002.

BRADFORD, M.A.; TORDOFF, G.M.; BLACK, H.I.J.; COOK, R.; EGGERS, T.; GARNETT, M.H.; GRAYSTON, S.J.; HUTCHESON, K.A.; INESON, P.; NEWINGTON, J.E.; OSTLE, N.; SLEEP, D.; STOTT, A.; HEFIN JONES, T. Carbon dynamics in a model grassland with functionally different soil communities. Functional Ecology, v.21, p.690-697, 2007. DOI: 10.1111/j.13652435.2007.01268.x.

CARDOSO, E.J.B.N.; VASCONCELLOS, R.L.F.; BINI, D.; MIYAUCHI, M.Y.H.; SANTOS, C.A. dos; ALVES, P.R.L.; PAULA, A.M. de; NAKATANI, A.S.; PEREIRA, J. de M.; NOGUEIRA, M.A. Soil health: looking for suitable indicators. What should be considered to assess the effects of use and management on soil health? Scientia Agricola, v.70, p.274-289, 2013. DOI: $10.1590 / \mathrm{S} 0103-90162013000400009$.

CASÃO JUNIOR, R.; ARAÚJO, A.G. de; LLANILLO, R.F. Plantio direto no Sul do Brasil: fatores que facilitaram a evolução do sistema e o desenvolvimento da mecanização conservacionista. Londrina: IAPAR, 2012. 77p.

CORREIA, M.E.F.; AQUINO, A.M. de; AGUIAR-MENEZES, E. de L. Aspectos ecológicos dos Isopoda terrestres. Seropédica: Embrapa Agrobiologia, 2008. 23p. (Embrapa Agrobiologia. Documentos, 249).

EIVAZI, F.; TABATABAI, M.A. Glucosidases and galactosidases in soils. Soil Biology and Biochemistry, v.20, p.601-606, 1988. DOI: 10.1016/0038-0717(88)90141-1.

HARASIM, E.; GAWEDA, D.; WESOLOWSKI, M.; KWIATKOWSKI, C.; GOCOL, M. Cover cropping influences physico-chemical soil properties under direct drilling soybean. Acta Agriculturae Scandinavica, Section B - Soil and Plant Science, v.66, p.85-94, 2016. DOI: 10.1080/09064710.2015.1066420.

HERNANDES, R.; CAZETTA, J.O. Método simples e acessível para determinar amônia liberada pela cama aviária. Revista
Brasileira de Zootecnia, v.3, p.824-829, 2001. DOI: 10.1590/ S1516-35982001000300030.

JÄGGI, W. Die Bestimmung der $\mathrm{CO}_{2}$-Biulding als $\mathrm{Ma} \beta$ der bonbodenbiologischen Aktivität. Schwiez Landwirtschaft Forchung Band, v.314, p.317-380, 1976.

MENDES, S.M.; SANTOS, J.; FREITAS, H.; SOUSA, J.P. Assessing the impact of understory vegetation cut on soil epigeic macrofauna from a cork-oak Montado in South Portugal. Agroforestry Systems, v.82, p.139-148, 2011. DOI: 10.1007/ s10457-010-9358-z.

MILCU, A.; PARTSCH, S.; SCHERBER, C.; WEISSER, W.W.; SCHEU, S. Earthworms and legumes control litter decomposition in a plant diversity gradient. Ecology, v.89, p.1872-1882, 2008. DOI: $10.1890 / 07-1377.1$.

MONTECCHIA, M.S.; CORREA, O.S.; SORIA, M.A.; FREY, S.D.; GARCÍA, A.F.; GARLAND, J.L. Multivariate approach to characterizing soil microbial communities in pristine and agricultural sites in Northwest Argentina. Applied Soil Ecology, v.47, p.176-183, 2011. DOI: 10.1016/j.apsoil.2010.12.008.

PARRA, J.R.P.; PANIZZI, A.R.; HADDAD, M.L. Índices nutricionais para medir consumo e utilização de alimento por insetos. In: PANIZZI, A.R.; PARRA, J.R.P. (Ed.). Bioecologia e nutrição de insetos: base para o manejo integrado de pragas. Brasília: Embrapa Soja, 2009. p.37-90.

PAUL, B.K.; VANLAUWE, B.; HOOGMOED, M.; HURISSO, T.T.; NDABAMENYE, T.; TERANO, Y.; SIX, J.; AYUK, F.O. Exclusion of soil macrofauna did not affect soil quality but increased crop yields in a sub-humid tropical maize-based system. Agriculture, Ecosystems and Environment, v.208, p.75-85, 2015. DOI: 10.1016/j.agee.2015.04.001.

QIN, S.; HE, X.; HU, C.; ZHANG, Y.; DONG, W. Responses of soil chemical and microbial indicators to conservational tillage versus traditional tillage in the North China Plain. European Journal of Soil Biology, v.46, p.243-247, 2010. DOI: 10.1016/j. ejsobi.2010.04.006.

RABARY, B.; SALL, S.; LETOURMY, P.; HUSSON, O.; RALAMBOFETRA, E.; MOUSSA, N.; CHOTTE, J.-L. Effects of living mulches or residue amendments on soil microbial properties in direct seeded cropping systems of Madagascar. Applied Soil Ecology, v.39, p.236-243, 2008. DOI: 10.1016/j. apsoil.2007.12.012.

SANTOS, H.G. dos; JACOMINE, P.K.T.; ANJOS, L.H.C. dos; OLIVEIRA, V.A. de; LUMBRERAS, J.F.; COELHO, M.R.; ALMEIDA, J.A. de; CUNHA, T.J.F.; OLIVEIRA, J.B. de. Sistema brasileiro de classificação de solos. 3.ed. rev. e ampl. Brasília: Embrapa, 2013. 353p.

SILESHI, G.; MAFONGOYA, P.L.; CHINTU, R.; AKINNIFESI, F.K. Mixed-species legume fallows affect faunal abundance and richness and $\mathrm{N}$ cycling compared to single species in maize-fallow rotations. Soil Biology and Biochemistry, v.40, p.3065-3075, 2008. DOI: 10.1016/j.soilbio.2008.09.007.

SILVA, A.P.; BABUJIA, L.C.; FRANCHINI, J.C.; SOUZA, R.A.; HUNGRIA, M. Microbial biomass under various soil- and crop-management systems in short- and long-term experiments in Brazil. Field Crops Research, v.119, p.20-26, 2010. DOI: 10.1016/j.fcr.2010.06.012. 
SILVA, R.F. da; AQUINO, A.M. de; MERCANTE, F.M.; GUIMARÃES, M. de F. Populações de oligoquetos (Annelida: Oligochaeta) em um Latossolo Vermelho submetido a sistemas de uso do solo. Ciência Rural, v.36, p.673-677, 2006. DOI: 10.1590/ S0103-84782006000200051.

SILVA, R.F. da; TOMAZI, M.; PEZARICO, C.R., AQUINO, A.M. de; MERCANTE, F.M. Macrofauna invertebrada edáfica em cultivo de mandioca sob sistemas de cobertura do solo. Pesquisa Agropecuária Brasileira, v.42, p.865-871, 2007. DOI: 10.1590/ S0100-204X2007000600014.

SILVA, R.F. da; CORASSA, G.M.; BERTOLLO, G.M.; SANTI, A.L.; STEFFEN, R.B. Fauna edáfica influenciada pelo uso de culturas e consórcios de cobertura do solo. Pesquisa Agropecuária Tropical, v.43, p.130-137, 2013. DOI: 10.1590/ S1983-40632013000200001.

TABATABAI, M.A.; BREMNER, J.M. Assay of urease activity in soil. Soil Biology and Biochemistry, v.4, p.479-487, 1972. DOI: 10.1016/0038-0717(72)90064-8.
TIEMANN, L.K.; GRANDY, A.S.; ATKINSON, E.E.; MARINSPIOTTA, E.; MCDANIEL, M.D. Crop rotational diversity enhances belowground communities and functions in an agroecosystem. Ecology Letters, v.18, p.761-771, 2015. DOI: 10.1111/ele.12453.

TORRES, J.L.R.; PEREIRA, M.G.; RODRIGUES JUNIOR, D.J.; LOSS, A. Production, decomposition of residues and yield of maize and soybeans grown on cover crops. Revista Ciência Agronômica, v.46, p.451-459, 2015. DOI: 10.5935/1806-6690.20150026.

VANCE, E.D.; BROOKES, P.C.; JENKINSON, D.S. An extraction method for measuring soil microbial biomass C. Soil Biology and Biochemistry, v.19, p.703-707, 1987. DOI: 10.1016/00380717(87)90052-6.

ZIECH, A.R.D.; CONCEIÇÃO, P.C.; LUCHESE, A.V.; BALIN, N.M.; CANDIOTTO, G.; GARMUS, T.G. Proteção do solo por plantas de cobertura de ciclo hibernal na região Sul do Brasil. Pesquisa Agropecuária Brasileira, v.50, p.374-382, 2015. DOI: 10.1590/S0100-204X2015000500004.

$\overline{\text { Recebido em } 31 \text { de agosto de } 2015 \text { e aprovado em } 3 \text { de fevereiro de } 2016}$ 